\title{
Numerical Evaluation of Cushioning Pressure in Water Entry of Rigid Bodies
}

\author{
Mojtaba Barjasteh ${ }^{1}$, Hamid Zeraatgar ${ }^{2 *}$ \\ ${ }^{1}$ Faculty of maritime engineering; barjasteh@aut.ac.ir \\ ${ }^{2}$ Faculty of maritime engineering; hamidz@aut.ac.ir
}

\section{ARTICLE INFO}

Article History:

Received: 11 Mar. 2017

Accepted: 21 Sep. 2017

\section{Keywords:}

Water entry

Air cushion

Cushioning effect

\begin{abstract}
Effect of air cushion layer right before impact of a rigid body onto water surface has been investigated in this paper. The study is mainly focused on evaluation of cushioning pressure and the resulting free surface elevation. The air flow is assumed to be an irrotational flow which is governed by Laplace equation. The air problem and the resulting response of the water free surface are supposed to be weakly coupled because of very low air pressure. Integral equation for each medium has been numerically solved separately using boundary element method. The problem is assumed to be unsteady with a constant body speed. The numerical results have been also compared with analytical method which shows a fair agreement. Results show that the geometry of impacting body and particularly its bluntness are the primary affecting parameter which can dramatically influence the free surface profile and air pressure. Such a behavior has been observed for two different geometries, ellipse and wedge section, having identical breadth.
\end{abstract}

\section{Introduction}

Water impact has many practical applications in marine engineering such as evaluation of slamming forces, seaplane design, wave loads on offshore structures, design of surface piercing propeller, analysis of motion in waves, etc. Considerable studies have been made on this topic which are mainly concentrated on prediction of the impact force. A variety of methods have been employed to implement the problem, ranging from pure numerical to entirely experimental method, but most of them neglect the effect of air gap right before impact for simplicity. However, the cushioning problem should be essentially considered in particular problems such as droplets and blunt body impacts. Generally, the air cushion layer affect the entry problem at least in three ways. The first one is to reduce the impacting speed due to well-known air drag force particularly right before impact. So, the resulting impact pressure is also decreased. The second effect of the air gap or air cushioning layer is to disturb the free surface. Neglecting air cushion effects means that the free surface is remained extremely planar at the moment of impact. However, presence of air cushion causes an induced pressure over the free surface. The free surface responds to this pressure to balance the forces along the air-water interface and thus it is deformed. The elevated free surface causes a multi-points contact rather than a single point contact $[1,2,3]$. This is schematically illustrated in Figure 1. The induced air pressure may also deform the impacting body if it is liquid such as those happen in droplet impact on liquids or solids. The number of contact points changes the generated pattern of impact pressure in the water and its propagation $[4,5]$.

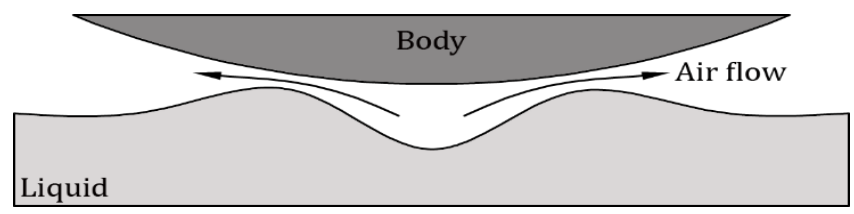

Figure 1. Exaggerated schematic view of escaping air flow and resulting double point contact

The third effect concerns entrapped air between the body and the elevated free surface. The contact area gradually increases between the body and the free surface profile as the body penetrates more into water. Therefore, the entrapped air is compressed at the initial stage of entry. Compressing the air can dissipate impact momentum. This dissipation reduces the slamming pressure which is experienced by the impacting body $[3,4,5]$. Depending on the volume of the entrapped air, the importance of this effect varies.

Study of air cushion can be employed in several engineering applications. An interesting case is droplet impact on liquid or solid surface. This topic has a wide range of applications in coating process, cooling and 
cleaning of surfaces, atomization, inkjet printing and raindrop dynamics. Cushioning effect is also important in marine vehicles such as air supported vehicles like SES and hovercrafts, lifting ultra-heavy buoyant structures, prediction of slamming force on the wet deck of catamarans, etc.

Most of cushioning characteristics are dependent on the volume, shape and location of entrapped air beneath the body. These parameters themselves are highly affected by air flow properties. So, the cushioning may show a random behavior depending on the problem. It can be shown that as bluntness of the body increases, the presence of air gap becomes more pronounced. So, it is expected that cushioning effect has a significant influence on a flat plate entry. Figure 2 illustrates a typical CFD simulation which is figured out by the Authors to show air entrapment before and after entry of a flat plate. This numerical simulation is carried out using the commercial CFD code, FLUENT. The solver employs a RANS algorithm coupling with the VOF model to evaluate the disturbance of free surface profile. The well-known dynamic mesh techniques controls the motion of grid nodes based on the rigid body dynamics. It should be mentioned that in most of applications, the cushioning corresponds to initial stage of penetration, ranging from nanoseconds to milliseconds.

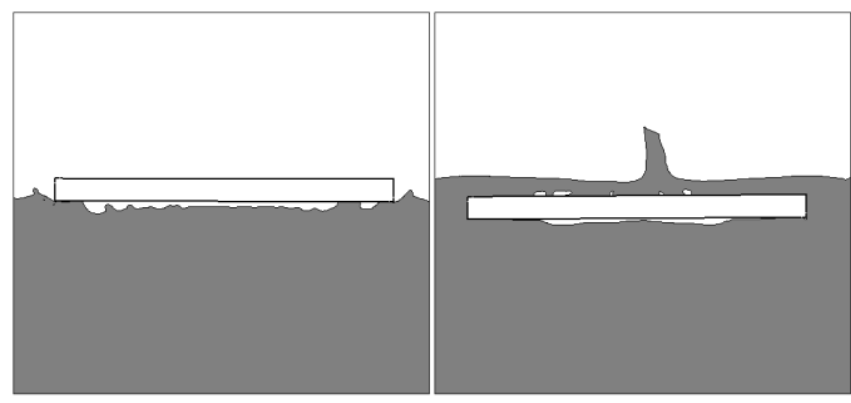

Figure 2. CFD simulation of air entrapment before and after impact of a flat plate

Air cushion problem is mostly studied for investigation of droplet impacts. Study of air cushion has a great impact on the drops and bubbles dynamics. Almost after a century past from pioneer study of droplet impact made by Worthington [6], the presence of air layer has been continuously investigating. Some of recent developments include Thoroddsen, et al. [7], Marston, et al. [8], Hicks and Purvis [4] and Tran, et al. [5] which showed different characteristics of air bubble formation and its rapture in different impact problem. Particular interest of the present research is on the water impact of larger solid bodies onto water. The impact pressure may be roughly predicted by traditional theories presented by Von Karman [9] and Wagner [10]. These theories and almost all other water entry theories neglect presence of air cushion for the sake of simplicity. However, it should be considered in some particular problems.
At the first investigation, Chuang [11] and Nethercote, et al. [12] experimentally showed that the pressure recorded at the apex of a roughly parabolic or small deadrise angle bodies has an oscillation behavior. They reported that this oscillation is not an electronic noise. They experimentally revealed that the presence of cushioning air layer is the source of this oscillation. Several later experimental researches such as those conducted by Okada and Sumi [13], Ermanyuk and Ohkusu [14] and also Huera-Huarte , et al. [15] studied dependency of the impact pressure to the cushioning layer by conducting different impact tests. The most important parameter of these research is geometry of impacting bodies and their bluntness. They generally confirmed that reducing deadrise angle of wedges and also increasing bluntness of bodies can dramatically affect the magnitude, pattern and spatial distribution of impact pressure due to presence of cushioning effect and resulting air entrapment.

Different analytical researches investigated the cushioning problem mathematically. The first analytical model proposed by Verhagen [1]. He employed a simple one dimensional model for escaping air flow between a flat plate and elevated free surface. The gap layer was modeled by a channel flow in which the air flow governs by practical compressible gas dynamics formulation. He supposed that the air flow is choked at the air gap and therefore the air flow speed would be equal to local speed of sound. Cushioning pressure as well as escaping air speed were both reported in this study. Lewison and Maclean [16] and Lewison [17] developed the Verhagen model [1] by conducting series of experiments including flat plate impacts and showed that the Verhagen model [1] was in a good agreement with experimental results. In an independent research, Asryan [18] also recommended a mathematical model to investigate the problem based on a small perturbation analysis. He assumed that the free surface deformation is small in comparison with the characteristic length of the body, and presented the equation of viscous squeeze film and Reynolds equation for flow in a channel with moving walls. His theoretical model provided more details of the problems rather than Verhagen's model. The presented model could not accurately capture the free surface profile and just available for initial stage of surface deformation. The model estimates pressure distribution in air flow. Wilson [2] employed non-dimensional form of two dimensional Navier-Stokes equations to propose a pair of coupled integral and differential equation for modeling of the problem. The results of this research included free surface profile and air cushioning pressure both in non-dimensional forms. He used different mathematical parameters to make the physical parameters non-dimensional. This made the proposed model impractical in engineering applications. Hicks and Purvis [3] further developed the Wilson's model and presented a more accurate model for three 
dimensional impact. They could remove drawbacks of the previous model. They employed a viscous lubrication equation to model the dynamics of air flow in cushioning layer. They first mathematically investigated the volume of air entrapment and presented a dimensional analysis for this volume and for different body geometries. Hicks, et al. [19] validated the proposed theory by conducting a set of experiments to study the effect of air-trapping by a rigid sphere impacting on a free surface of the liquid of infinite depth. Formation of entrapped air was captured by a high speed camera. They showed that the experimental and theoretical results are in a good agreement. Instantaneous air pressure and free surface profile are the main output of this research.

The present study investigates the air cushion effect in water entry of blunt rigid bodies in which the cushioning pressure cannot be neglected. Both air and water flows are assumed to be irrotational and governed by Laplace equation. Solution of the governing equation is numerically carried out employing boundary element method with nonlinear boundary conditions. Instantaneous free surface profile as well as air pressure are evaluated. The coupling mechanism between air and water flow is figured out via an artificial boundary separation for the first time. This technique can significantly simplify the problem formulation and its numerical implementation. Since the BEM is used to solve the problem, the recommended model provides a rapid method to solve cushioning problem with any arbitrary impacting geometry. Additionally, this model may be employed to simulate droplet impact onto other droplets or onto liquid or solid surface. The numerical results are compared with other similar research.

\section{Formulation}

It is assumed that the impacting body is rigid and water depth is infinite. The rigid body approaches the water surface with a constant vertical speed, $V_{i}$. For high speed impact of blunt bodies, one can neglect free surface tension. This results in an inertial dominant flow regime in the cushioning layer. Furthermore, air and water flows are rapid strain flows which inertial forces are dominant and the viscous effects are neglected for high speed impact. Thus, both flows are assumed to be irrotational which are governed by Laplace equation.

$$
\frac{\partial^{2} \phi}{\partial x^{2}}+\frac{\partial^{2} \phi}{\partial y^{2}}=0
$$

where $\phi$ is velocity potential. Although geometry of the body can have any arbitrary shapes, it is supposed to be symmetric for the sake of simplicity. This assumption makes both air and water flows symmetric and consequently simplifies the problem as a half domain which significantly reduces computational time.
Figure 3 illustrates the physical and computational domains of the problem. The problem is divided into two weakly coupled problem of the air flow and the water flow. The air problem is bounded with surfaces of $S_{1}, S_{2}, S_{3}, S_{4}, S_{b}$ and $S_{a}$ where $S_{b}$ and $S_{a}$ are external surface of body and free surface profile, respectively. Similarly, boundaries of the water problem includes $S_{6}$, $S_{7}, S_{8}$ and $S_{w}$. The free surface profile is artificially divided into two different surfaces $S_{a}$ and $S_{w}$ with the same dynamics. This is the primary technique of the simulation in this study. The air flow and the water are coupled through the motion of their interface.

Figure 4 depicts typical response of these surfaces at different time steps.

Boundary conditions of the problem should be specified for solution of the boundary value problem in both air and water flows. For the air far field boundary condition is applied for boundaries $S_{3}$ and $S_{4}$ as follow:

$$
\phi_{a}(\infty, t)=0
$$
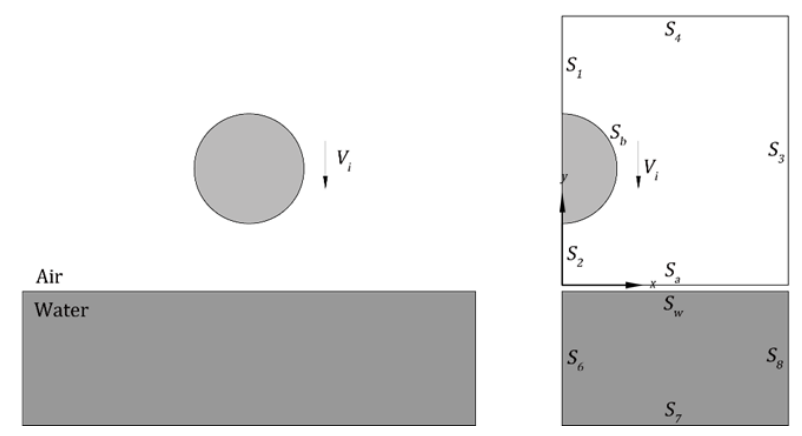

Figure 3. Physical and computational domain of the problem
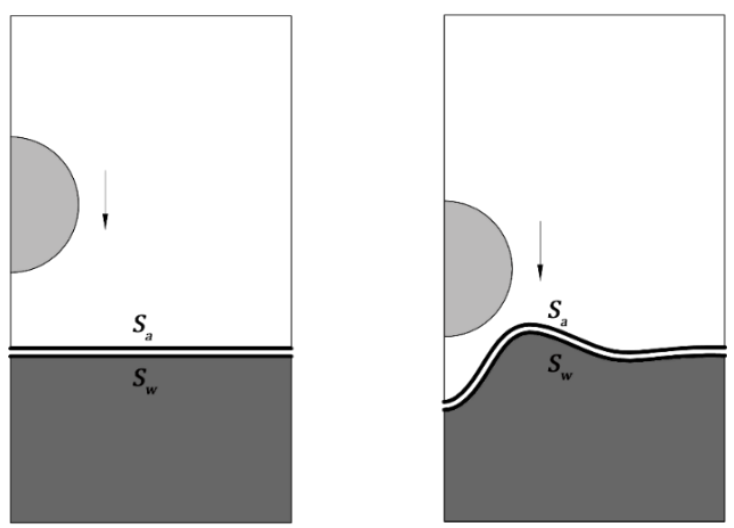

Figure 4. Schematic view of artificially divided surfaces in two different time steps

Subscript $a$ indicates air flow. No flux boundary condition is also applied for external surface of the body, $S_{b}$ and symmetric boundaries $S_{1}$ and $S_{2}$ as follows:

$$
\frac{\partial \phi_{a}}{\partial n}=\hat{n} \cdot \vec{V}
$$

where $\hat{n}$ is the normal vector of the body geometry pointing outward. The remaining boundary is free surface boundary, namely $S_{a}$ in the air problem. The specified boundary conditions on this surface consist of well-known nonlinear kinematic and dynamic free 
surface boundary conditions. Kinematic free surface boundary condition presented as follows.

$$
\frac{\partial \phi_{a}}{\partial y}=\frac{D \eta(x, t)}{D t}=\left[\frac{\partial \eta(x, t)}{\partial t}+\frac{\partial \phi_{a}}{\partial x} \frac{\partial \eta}{\partial x}\right]_{y=\eta}
$$

here $\eta(x, t)$ is free surface profile. Dynamic boundary condition balances the applied forces on the interface.

$$
\frac{\partial \phi_{a}}{\partial t}+\frac{1}{2}|\nabla \phi|^{2}=g \eta-\frac{\sigma}{\rho_{a}} \kappa(x, t)-\frac{P_{a}}{\rho_{a}}
$$

where $g, \sigma$ and $\kappa$ are gravitational acceleration, surface tension at the air-water interface and curvature of free surface, respectively. This curvature is a function of spatial derivatives of free surface profile.

$$
\kappa(x, t)=\left[\left|\frac{\partial^{2} \eta}{\partial x^{2}}\right| \cdot\left(1+\frac{\partial \eta}{\partial x}\right)^{-\frac{3}{2}}\right]_{y=\eta}
$$

Equation 6 includes both gravity effects and surface tension. Considering these parameters increases the accuracy of modeling particularly at initial stage of surface deformation.

For the water problem, far field and symmetric boundary conditions are applied for boundaries $S_{8}$ and $S_{6}$, respectively. Wall boundary condition is also determined for the water bed.

$$
\frac{\partial \phi_{w}}{\partial n}=\hat{n} \cdot \vec{V}_{w}=\hat{n}_{x} u_{w}+\hat{n}_{y} v=0
$$

Similar kinematic and dynamic boundary condition should be applied for the boundary $S_{w}$ with substituting appropriate properties of water instead of the air.Now the governing equations of the problem and their corresponding boundary conditions are known. Air and water problems are coupled to each other by the pressure at their interface.

Different researches showed that the following scaling for the entrapped air bubble volume was found at this condition.

$$
\delta=V_{e} / V_{b} \approx S t^{-\frac{4}{3}}
$$

where, $\delta$ is the air bubble volume normalized by the drop volume and $S t$ is the Stokes number, which is defined as

$$
S t=\frac{\rho_{l} R U}{\mu_{g}}
$$

here $\rho_{l}$ is the liquid density, $R$ is the droplet or body radius, $U$ is its impact velocity and $\mu_{g}$ is the viscosity of the surrounding gas, in this case air. In case of noncircular body section, $R$ is the equivalent curvature of bottom section of the body. The Stokes number represents the competing effects of the viscous force of the draining air film and the inertial force of the liquid, which ultimately determine the air bubble volume. The same scaling was found experimentally for impact of a sphere onto a pool [3] and a drop onto a pool [4,19]. When surface tension effects become important, the scaling must be modified to include the effect of the Laplace pressure as moves towards the capillary regime [20].

\section{Numerical implementation}

Dynamics of the problem in air and water flows are governed by Laplace equation. This elliptic partial differential equation is well suited to be numerically solved using boundary element method. This method is widely employed for solution of the Laplace equation especially in water entry and related problems such as employed by Yousefnezhad and Zeraatgar [21]. Numerical solution is started by mesh generation on the desired boundaries. Special cares should be taken for discretization. The BEM is very sensitive to element size in area close to sources distributed on the boundaries such as intersection of free surface and body surface, $S_{2}$ (see Figure3). This sensitivity is highly dependent on the length of the boundary $S_{2}$. Once the body gets very close to the free surface, the length of the boundary $S_{2}$ is very small and the body as the source of potential perturbation is very close to the sources distributed on the free surface. Thus the element size is necessarily very small on both the body and free surface to adequately capture the potential gradient. Figure 5 illustrates the boundaries for an ellipse entry when it is close to the free surface. To obtain a reasonable mesh density at the interface, spatial discretization on the free surface boundary is carried out using geometric progression. This provides finer elements on the origin and coarse elements at the physical infinity to control the computational time and performance of the solver, simultaneously.

The numerical simulation is carried out in different time steps. At each time step, the problem is first solved for the air flow. Then the obtained results are employed to solve the water flow. The primary air flow characteristic which is used for water problem is the pressure distribution on the free surface. Since, the flows are not solved simultaneously, the modeling may be called as a weakly coupled problem. Boundary conditions of the problem remain unchanged during all time steps. However, the boundary conditions on $S_{a}$ and $S_{w}$ are not the same in all time steps.

It can be shown that the elevation of free surface due to presence of cushioning pressure is important when the body is very close to the free surface. Therefore, the elevation at initial stage of the body motion may be neglected, say the first time step $t_{0}$. So, it can be formulated by substituting the free surface, $S_{a}$, with a rigid wall.

$$
\frac{\partial \phi_{a}}{\partial n}=0 @ t=t_{0}
$$




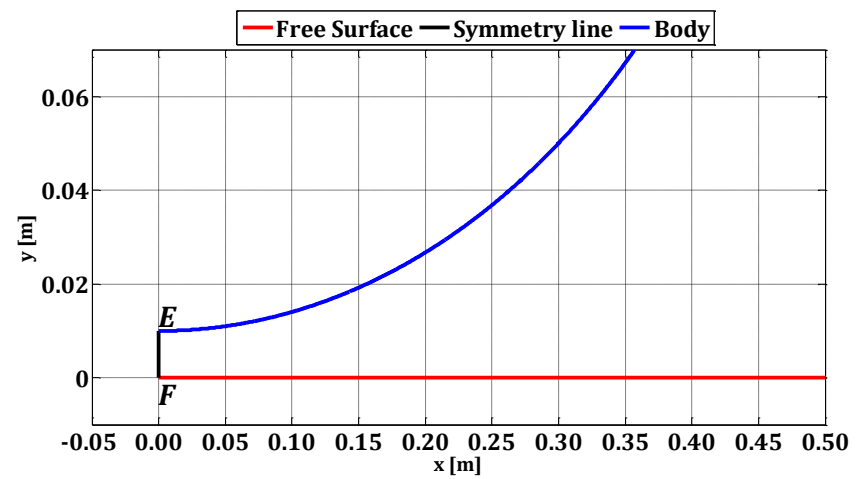

(a)

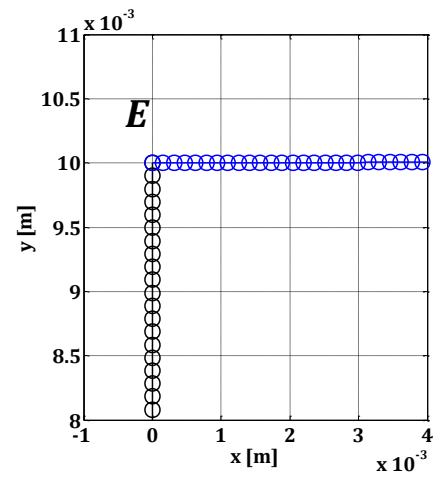

(b)

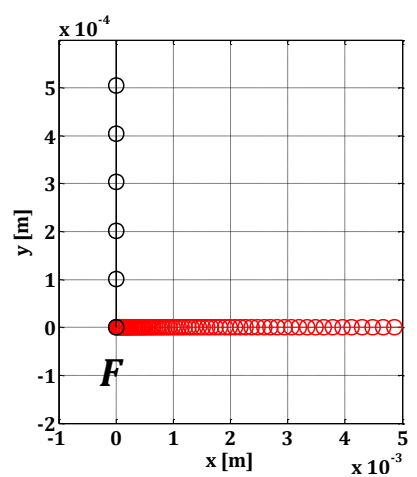

(c)
Figure 5. Mesh density on different boundary for an ellipse for with $\zeta=3$ and $V_{0}=1 \mathrm{~m} / \mathrm{s}$, (a) half domain of the problem, (b) magnification of the mesh density at the intersection point of the body and the symmetry line, $(c)$ magnification of the mesh density at the intersection point of the symmetry line and the free surface.

The air problem can now be solved using boundary element method at time $t_{0}$ with pre-defined boundary conditions. According to these conditions, this problem is classified as a mixed Dirichlet-Neumann problem. It can be shown that applying second Green identity for a distributed source, $F$, on the boundary yields [22]:

$$
\phi(P)=-\int_{\Gamma}\left[\lambda(F, q) \frac{\partial \phi(q)}{\partial n_{q}}-\phi(q) \frac{\partial \lambda(F, q)}{\partial n_{q}}\right] d s_{q}
$$

where, $q$ is an arbitrary integration point on the boundary and $\lambda$ is the fundamental solution of Laplace equation as follows [22].

$$
\begin{aligned}
& \lambda=\frac{1}{2 \pi} \ln r(F, q) \\
& \frac{\partial \lambda}{\partial n_{q}}=\frac{1}{2 \pi} \frac{\cos (\operatorname{angle}(r, n))}{r}
\end{aligned}
$$

and $r=|q-F|$ is the distance vector. The potential integral equation can be discretized in following format assuming constant distribution of potential on each element [22]:

$$
-\frac{1}{2} \phi^{i}+\sum_{j=1}^{N} \widehat{H}_{i j} \phi^{j}=\sum_{j=1}^{N} \widehat{G}_{i j} \frac{\partial \phi^{j}}{\partial n}
$$

where, $H$ and $G$ are influence coefficients and $N$ is the total number of elements. Once the problem at time step $t_{0}$ is solved using BEM, the potential on the free surface, $\phi_{a 0}$, is known on $S_{a}$. At the next time step, increased by $\Delta t$, the new position of the body is updated and the problem is again solved with previous boundary conditions but with new position of the body. The new potentials on $S_{a}$, say $\phi_{a 1}$, is obtained at time step $t_{1}=t_{0}+\Delta t$. So, air pressure due to vertical motion of the body can be evaluated using unsteady Bernoulli equation on $S_{a}$.

$$
P_{a}=-\rho_{a}\left[\frac{\partial \phi_{a}}{\partial t}+\frac{1}{2}\left|\nabla \phi_{a}\right|^{2}+g \eta_{a}\right]
$$

where, $\eta_{a}$ is instantaneous profile of $S_{a}$ which is vanished in the unsteady Bernoulli equation because of rigid wall assumption. Furthermore, since the free surface is not permitted to be deformed, the surface tension is also disappeared. The only unknown variable is $\partial \phi / \partial t$. For reasonably small enough $\Delta t$ in all time steps, the following approximation is readily available using Taylor expansion.

$$
\frac{D \phi_{a}}{D t} \approx \frac{\Delta \phi_{a}}{\Delta t}=\frac{\phi_{a 1}-\phi_{a 0}}{t_{1}-t_{0}}
$$

And

$$
\frac{\partial \phi_{a}}{\partial t}=\frac{D \phi_{a}}{D t}-\left|\nabla \phi_{a}\right|^{2} \approx \frac{\Delta \phi_{a}}{\Delta t}-\left|\nabla \phi_{a}\right|^{2}
$$

It is worth noting that the derivatives of the potential are evaluated using a forward time differencing scheme. Thus, the obtained air pressure corresponds to the first time step.

Now, solution of the air problem at the first time step is figured out. Using known values of potential and its derivatives on the free surface as well as the obtained air pressure, numerical solution of water problem is started. The water problem can be also solved using BEM by considering pre-defined boundary conditions. From the kinematic free surface boundary condition the normal derivative of water potential is vanished at time steps $t_{0}$ and $t_{1}$ on $S_{w}$.

$$
\frac{\partial \phi_{w}}{\partial n}=0
$$

Once the numerical solution is carried out, water velocity potentials are known on $S_{w}$ which may be zero at these time steps. At the next time step, $t_{2}$, there is no need to assume the free surface is rigid any more. Using dynamic free surface boundary condition, Bernoulli equation can be written on free surface for water problem at time step $t_{1}$ where the free surface elevation is yet zero. The only unknown parameter, $\partial \phi / \partial t$, is evaluated as follows.

$$
\frac{\partial \phi_{w}}{\partial t}=-\left(\frac{P_{a}}{\rho_{w}}+\frac{1}{2}\left|\nabla \phi_{w}\right|^{2}\right)
$$

Again using a forward time differencing scheme, the new values of potentials at the next time step, $t_{2}$, can be computed. 


$$
\phi_{w 2}=\Delta t\left(\frac{\partial \phi_{w}}{\partial t}+\left|\nabla \phi_{w}\right|^{2}\right)+\phi_{w 1}
$$

The potential on $S_{w}$ is now known at time step, $t_{2}$. The normal derivative of this potential is evaluated using boundary element method. The free surface profile, $\eta_{w}$, can be updated using the known values of normal and tangential derivatives of the potential at time step, $t_{2}$ based on kinematic and dynamic free surface boundary conditions.Velocity components can be estimated using differencing formula. However, for more precise results, they are obtained using following boundary integrals [22]. A typical result of evaluated components of fluid particles on the air-water interface is depicted in Figure 6.

$$
\begin{aligned}
& \frac{\partial \phi}{\partial x}=-\int_{\Gamma}\left[\frac{\partial \lambda}{\partial x} \frac{\partial \phi}{\partial n}-\phi \frac{\partial}{\partial x}\left(\frac{\partial \lambda}{\partial n}\right)\right] d s \\
& \frac{\partial \phi}{\partial y}=-\int_{\Gamma}\left[\frac{\partial \lambda}{\partial y} \frac{\partial \phi}{\partial n}-\phi \frac{\partial}{\partial y}\left(\frac{\partial \lambda}{\partial n}\right)\right] d s
\end{aligned}
$$

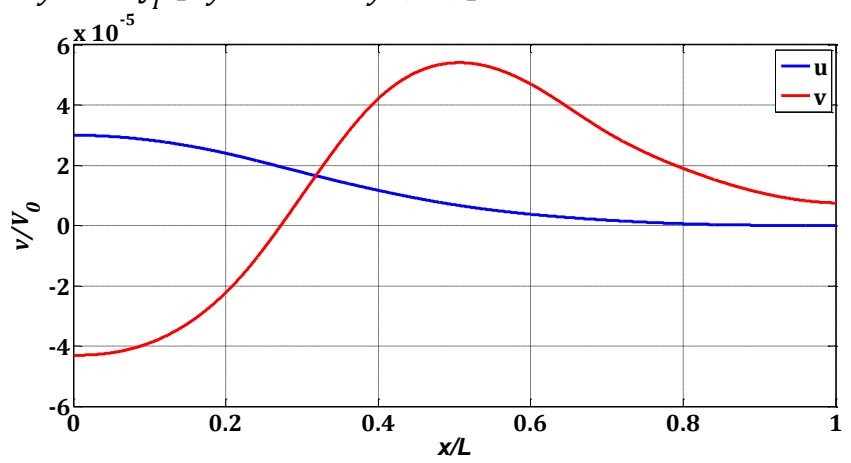

Figure 6. Typical non-dimensional horizontal and vertical velocity of the fluid particles on the free surface

At the current time step, the water problem is solved but the air problem is not solved, yet. The air flow can be solved after updating the new position of the body with the known values of $\phi_{n a}=\phi_{n w}$ and $\eta_{a}=\eta_{w}$. After solving the problem, the new values of the air pressure over free surface are obtained and solution of the problem can be continued for next time step. Coupling of the air and water problem at the second time step is very sensitive to specified boundary conditions on boundaries $S_{a}$ and $S_{w}$ which are artificially separated. Since the flows are irrotational, the boundary condition on the free surface is set to free slip condition. In other words, tangential derivatives of the air and water potentials, $\phi_{s}$, are not necessarily the same on the free surface. Therefore, the following kinematic free surface boundary condition readily concluded which must be satisfied on the free surface at each time step.

$$
\phi_{n a}=\phi_{n w}
$$

where, $\phi_{n a}$ and $\phi_{n w}$ are normal derivatives of the potentials in the air and water flows, respectively. Additionally, the dynamic free surface boundary condition implies that pressures on both sides of the free surface are equal. Including the surface tension for Capillary regime, the pressure balance yields:

$$
P_{s w}=P_{s a}-\sigma \frac{\partial^{2} \eta}{\partial x^{2}}
$$

where, $P_{S}$ is the pressure on the free surface and subscripts $a$ and $w$ indicate air and water flows, respectively. So, the numerical solution is fully accomplished.

All numerical methods have different limitation such as instability issues, truncation errors, computational time, etc. The most important issue in the boundary element method is its sensitivity to the boundary positions and their discretization. First, due to induced imaginary damping potential in BEM, the position of far filed boundaries must set adequately to remove this drawback. Additionally, the BEM solvers are very sensitive when the source of disturbance is very close to the boundaries. Such a condition arises in the present cushioning problem when the body is very close to the free surface. Thus, special attention should be paid on choosing the size of elements. Finally, the time step intervals are usually determined in a try and error algorithm. This is mainly due to sensitivity of the problem configuration to the temporal derivative of the potential. Thus, there is some limitation on simulation of the problem. Minimum air gap thickness which can be solved and simulated by the present code is about $\left(5 \times 10^{-4}\right) b$ where $\mathrm{b}$ is the body breadth.

Although, the water depth is assumed to be physically infinite, it can be controlled by surface $S_{6}$ for shallow water simulation. The present modeling can be easily employed for simulation of symmetric or fully three dimensional cushioning effects without any numerical difficulties.

\section{Results and discussions \\ 4.1 Validation}

Let starts with a circle instead of an ellipse for the sake of simplicity. The circle is approaching the free surface with constant and pure vertical speed. The solver evaluates the pressure distribution upon the free surface and elevation of the air-water interface at diameter of unity.

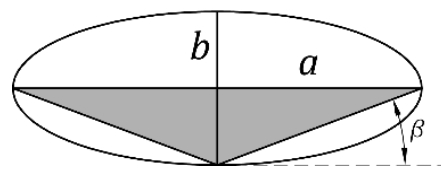

Figure 7. Geometry of the rigid bodies including an ellipse and its inscribed wedge.

Hicks and Purvis [19] had conducted a research on cylinder air cushion by analytical and experimental methods. The present numerical method is compared with Hicks and Purvis results for a circle having vertical velocity of $V_{0}=0.1 \mathrm{~m} / \mathrm{s}$ shown in

Figure 8 and

Figure 9. Generally, the comparison shows a reasonable agreement. The comparison shows that the present 
numerical method predicts a slightly higher peak pressure and faster pressure drop.

Figure 9 shows a comparison of the free surface profile calculated by present method with Hicks and Purvis method. The tendency as well as the profile is similar. The maximum deformation of free surface due to induced air pressure occurs at $x=0$ as expected. Interestingly, this deformation is very low for the circle under investigation.

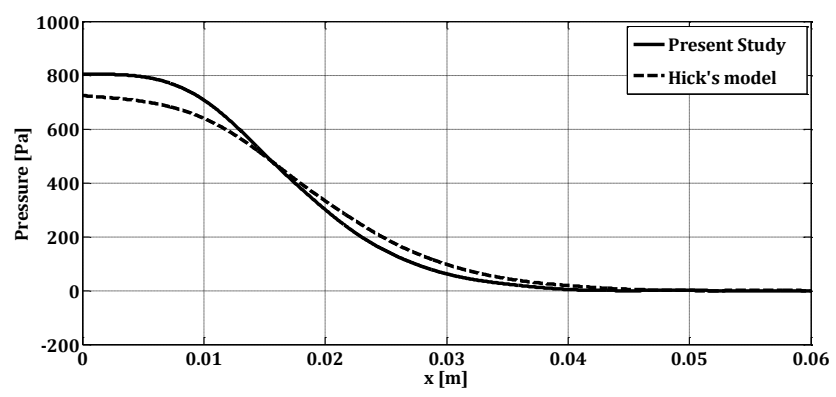

Figure 8. Comparison of air pressure on the free surface for a circle with $b=0.5$ and $V_{0}=0.1 \mathrm{~m} / \mathrm{s}$ with Hick's method.

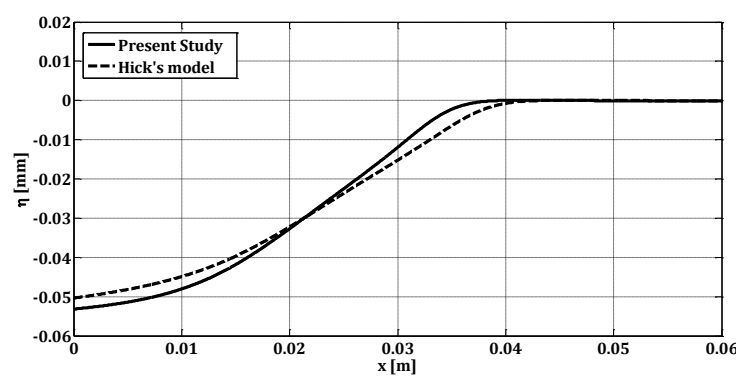

Figure 9. Comparison of elevated free surface profile for a circle with $b=0.5$ and $V_{0}=0.1 \mathrm{~m} / \mathrm{s}$ with Hick's method.

\subsection{Case studies}

The induced potential due to motion of a body on surrounding flow is highly affected by several parameters such as acceleration, geometry, speed and bluntness of the body. The two latter parameters are investigated in this study. Effects of bluntness are studied for an ellipse with different $\zeta=a / b$ from 1 to 10 , where $a$ and $b$ are the minor and major axes of the ellipse as illustrates in

Figure 7. The ellipse result is compared with the result of some corresponding inscribed wedge to study the effect of curvature of the body as well. The wedge breadth coincides with the major axis of the ellipses. This yields to an identical projected area in vertical direction. Thus, the projected area is fixed and the results are simply independent of this parameter. Clearly, the deadrise angle of the wedge is defined by $\beta=\tan ^{-1}(1 / \zeta)$. Major axis of the ellipse remains constant and equals to unity for all $\zeta$ ratios to retain the projected area unchanged. So, different body curvature is obtained by variation of minor axis.

The chosen geometry for study is $\zeta=3$ both for ellipse and wedge. The downward speed is $1 \mathrm{~m} / \mathrm{s}$. Time history of generated pressure are illustrated in
Figure 10 and

Figure 11 at five consecutive time steps. Although both geometries have the same width and the same speed, the magnitude of generated air pressures are totally different. This is mainly due to different bluntness of the bodies. In both cases, as the bodies get closer to the free surface, the peak pressure increases. So, the maximum pressure happens exactly at the horizontal origin. This is highly dependent on the capability of the solver to implement the thinner air gap. Thinner gaps results in different pressure distribution in which the maximum pressure is not placed at the origin. Due to different bluntness of the bodies, the rate of pressure drops is not the same.

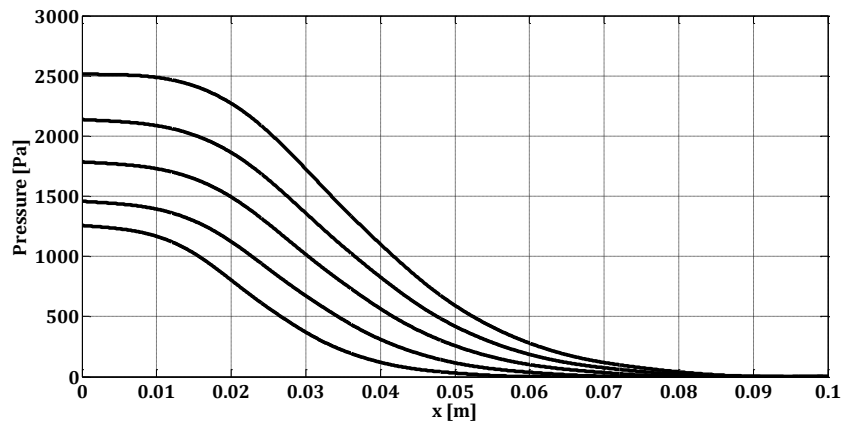

Figure 10. Air pressure over free surface for an ellipse and a wedge with $\zeta=3$ and $V_{0}=1 \mathrm{~m} / \mathrm{s}$ at different time steps with $\Delta \mathrm{t}=\mathbf{0 . 1 5} \mathrm{ms}$.

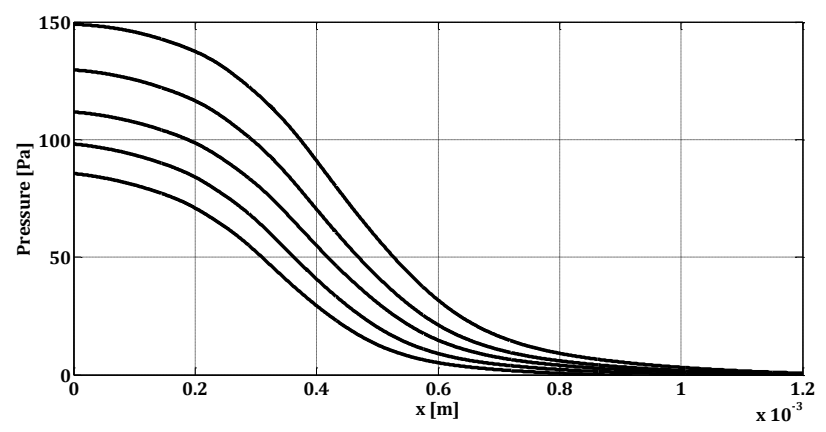

Figure 11. Air pressure over free surface for a wedge with $\zeta=$ 3 and $V_{0}=1 \mathrm{~m} / \mathrm{s}$ at different time steps with $\Delta \mathrm{t}=0.4 \mathrm{~ms}$.

Figure 12 depicts the elevated free surface profile for both bodies at last time. The profiles are different in both magnitudes and tendencies. The wedge induced free surface profile is extremely contracted region in comparison with the ellipse induced profile. This suggests that the cushioning pressure for the wedge is localized into a small region which may be neglected in comparison with the ellipse section. This may describe that why the cushioning effect is neglected for wedge section in real practice. 

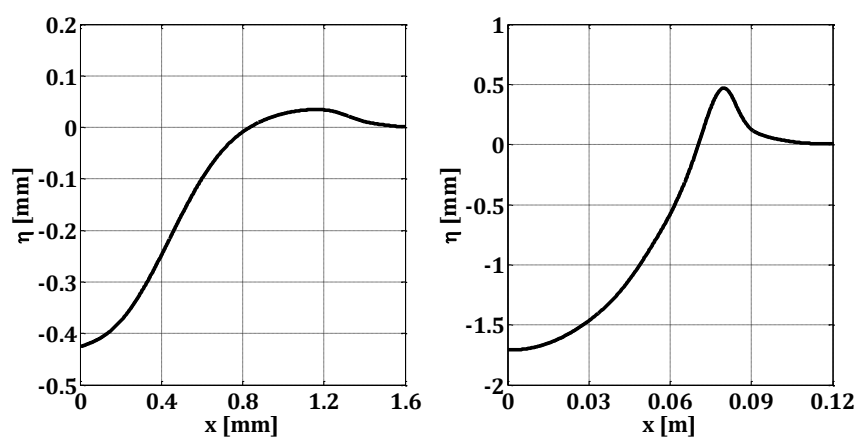

Figure 12. Elevated free surface profile for a wedge (left) and an ellipse (right) corresponding to the last time steps which are shown in figures 9 and 10.

Another interesting non-dimensional parameter is pressure coefficient which is here defined as follows.

$$
C_{P}=\frac{P_{\max }}{0.5 \rho_{a} V_{0}^{2}}
$$

where, $P_{\max }$ and $V_{0}$ are maximum air pressure over the free surface and vertical downward speed, respectively. This parameter can roughly compare the variation of pressure for different geometries. The pressure coefficient for the ellipse and the wedge sections at $\zeta=$ 3 are 5000 and 300, respectively. This comparison expeditiously shows the importance of the body bluntness on induced air pressure.

The disturbed free surface profiles due to cushioning pressure which are depicted in figures 8 and 11, show that the body would touch the free surface at two points. Therefore, the contact geometry would be a curve not a single point. Similarly, the contact area in threedimensional problem would be a surface similar to a donut. This multi-points contact can change the pattern of impact pressure of water and its propagation in the water which is usually neglected in most of water entry theories.

Since the bluntness of geometries significantly changes the air pressure, a parametric study is carried out on geometry bluntness, say $\zeta$.The air peak pressure is taken as a measure of merit. The breadth of the bodies is kept unchanged and $\zeta$ is increased incrementally. Then the problem is numerically solved for a set of geometries. Figure 13 compares the obtained peak pressure for both wedge and ellipse at the same value of $\zeta$.

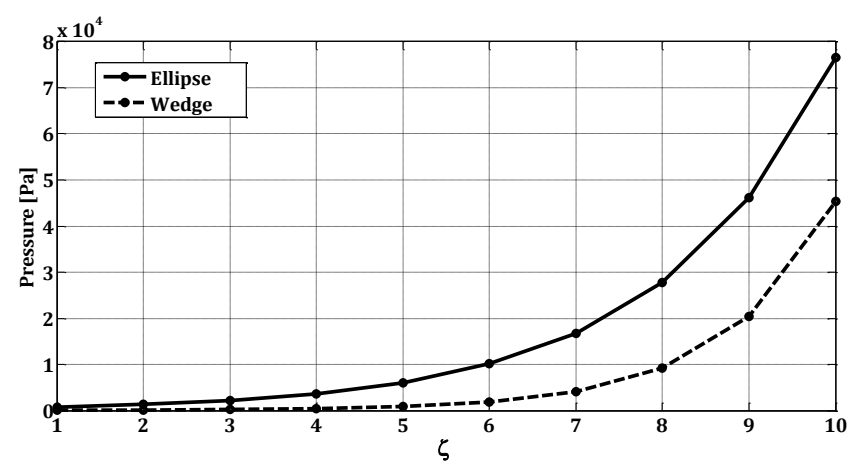

Figure 13. Variation of maximum cushioning pressure versus different $\zeta$ with the same values of $b$.
The calculated pressures exponentially increase as the bluntness increases. However, different physical phenomena prevent rising of air pressure to the estimated values in real practice. One of the most important one may be collapsing of the free surface profile. This results in a rapid pressure drop due to expansion of volume of air entrapment. Velocity reduction due dynamic of rigid body is another phenomenon which reduces air pressure.

Variation of peak pressures of both geometries shows an identical tendency. One can define a nondimensional pressure ratio as $\psi=P_{e} / P_{w}$ where $P_{e}$ and $P_{w}$ are the maxim peak pressure for the ellipse and the wedge sections, respectively.

Figure 14 illustrates variation of non-dimensional pressure ratio versus bluntness $\zeta$. This figure simply states that the non-dimensional pressure ratio, $\psi$, tends to a pre-specified value such as unity as the bluntness of the body goes to infinity. However, for $\zeta=10$ which both geometries seems to yield a flat plate, the wedge pressure is yet much less than that of the ellipse section. It is concluded that the cushioning pressure particularly for the very blunt body is very sensitive to the bluntness of the body.

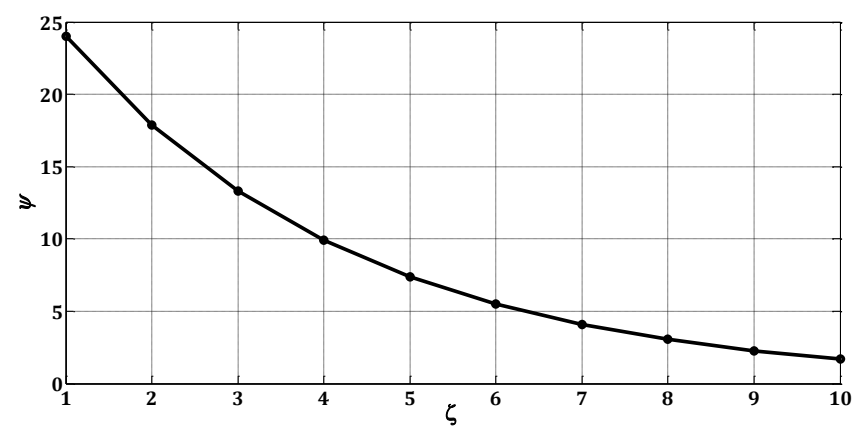

Figure 14. Variation of non-dimensional pressure ratio versus bluntness of the bodies

The bluntness of the body can also change distribution of the cushioning pressure especially location of the peak pressure. Error! Reference source not found. shows pressure distribution for an ellipse with $\zeta=6$ at two different time steps. As it is seen, the location of peak pressure gradually moves to the right side. Additionally, the pressure at the origin of horizontal axis decreases. This is primarily due to geometry of free surface profile where generates a high pressure necking section in cushioning layer. Furthermore, this behavior suggests that even a small perturbation in water surface can results in a totally different free surface profile. This is always the case in real practice. 


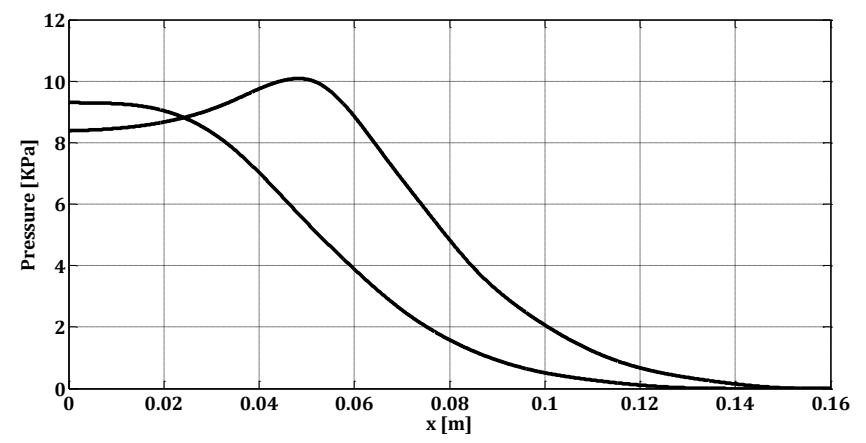

Figure 15. Pressure distribution for an ellipse with $\zeta=6$ at two consecutive time steps.

It should be noted that the air pressure are highly dependent on the width of the body and the air gap thickness. Additionally, the vertical speed of the body is assumed to be constant. Different speed changes the air pressure and resulting free surface profile in cushioning layer. Moreover, it is common in most of numerical studies to represent the results by nondimensional parameters. However, the results are intentionally stated in corresponding dimensions to provide a better physical understanding of the problem. Results of all numerical methods including BEM which employ discretization techniques are dependent on element size and mesh grid structure. Thus, mesh dependency analysis should be carried out for such methods. Element size in BEM method is generally determined by gradient of distributed potential. Figure 16 depicts a typical mesh dependency analysis for an ellipse section. The error is defined by $\left(P_{n+1}-P_{n}\right) / P_{n}$, where $P$ is the maximum air pressure and $\mathrm{n}$ indicates different mesh densities. The error value converges to less than \% 0.3 for the ellipse section. Similar mesh dependency analysis is also figured out for all case studies with different geometries and downward speed.

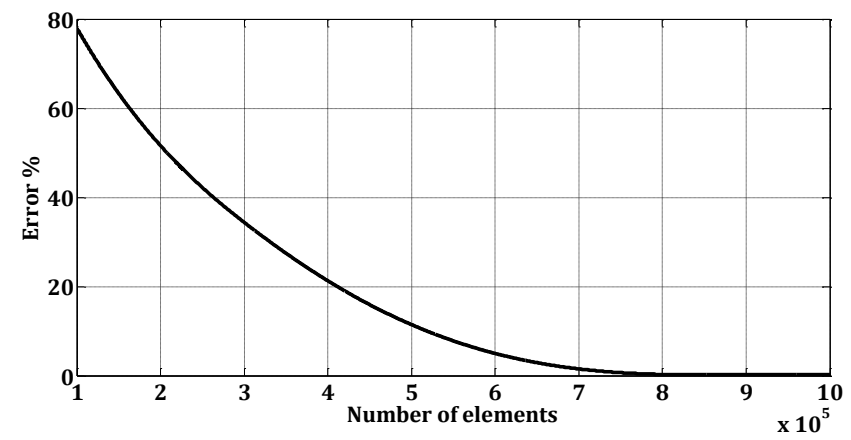

Figure 16. Typical mesh dependency analysis for an ellipse section

\section{Conclusions}

The cushioning problem is numerically investigated in this study using a weakly coupled modeling between the air and water flows. The solver employs a boundary element method to implement the problem. Generated cushioning air pressure as well as instantaneous free surface profile is evaluated. The obtained results show a fair agreement with analytical method. It is shown that characteristics of cushioning layer are dramatically affected by the bluntness of the body. The peak pressure is also evaluated for an inscribed wedge in the ellipse. The comparison of obtained results show that the effect of body curvature is vanished for large values of $\zeta$. Additionally, the numerical results prove that the single point contact never occurs in water entry problem especially for blunt bodies. Finally, the present study confirms that the cushioning effect can be neglected for wedges with relatively large deadrise angles while it must be considered for blunt bodies.

\section{Acknowledgements}

The authors would like to show their gratitude to Dr. Richard Purvis from university of East Anglia, UK, for his great comments on this paper.

\section{List of Symbols}

Below is the list of symbols which are employed in the present text.

$\begin{array}{ll}\rho_{a} & \text { Air density } \\ \rho_{w} & \text { Water density } \\ \sigma & \text { Air-Water surface tension } \\ P & \text { Pressure } \\ \phi & \text { Velocity potential } \\ \lambda & \text { Fundamental solution } \\ \eta & \text { Free surface profile } \\ \kappa & \text { Curvature } \\ \hat{n} & \text { Norma vector } \\ H_{i j}, G_{i j} & \text { Influence coefficients } \\ g & \text { Gravitational acceleration } \\ d s & \text { Length differential } \\ \Gamma & \text { Boundary identity } \\ a, b & \text { Dimensions of an ellipse } \\ \beta & \text { Deadrise angle } \\ \zeta & \text { Ellipse dimensional ratio } \\ \delta_{1}, \delta_{2} & \text { Non-dimensional length scales }\end{array}$

\section{References}

1- Verhagen, J.H.G., (1967), The impact of a flat plate on a water surface, Ship Res., Vol. 11, p.211-233.

2- Wilson, S.K., (1991), A mathematical model for the initial stages of fluid impact in the presence of a cushioning fluid layer, Engineering Mathematics, Vol. 25, p. 265-285. [DOI: 10.1007/BF00044334]

3- Hicks, P.D., Ermanyuk, E.V., Gavrilov, N.V. and Purvis, R., (2012), Air trapping at impact of a rigid sphere onto a liquid, Fluid Mechanics, Vol. 695, p. 310320. [DOI: $10.1017 / \mathrm{jfm} .2012 .20]$

4- Hicks, P. and Purvis, R., (2011), Air cushioning in droplet impacts with liquid layers and other droplets, physics of fluids, 23(6). [DOI: 10.1063/1.3602505] 
5- Tran, T., de Maleprade, H., Sun, C. and Lohse, D., (2013), Air entrainment during impact of droplets on liquid surfaces, Fluid Mechanics, Vol. 726. [DOI: 10.1017/jfm.2013.261]

6- Worthington, A.M., (1908), A Study of Splashes, Longmans, Green.

7- Thoroddsen, S.T., Etoh, T.G., Takehara, K., Ootsuka, N. and Hatsuki, Y., (2005), The air bubble entrapped under a drop impacting on a solid surface, Fluid Mechanics, Vol. 545, p. 203-212. [DOI: 10.1017/S0022112005006919]

8- Marston, J.O., VAKARELSKI, I.U., THORODDSEN, S.T., (2011), Bubble entrapment during sphere impact onto quiescent liquid surfaces, Fluid Mechanics, Vol. 680, p.660-670. [DOI: 10.1017/jfm.2011.202]

9- Von Karman, T., (1929), The impact on seaplane floats during landing, N.A.C.A.T.N. No. 321.

10- Wagner, H., (1931), Phenomena associated with impact and sliding on liquid surfaces, N.A.C.A. Translation 1366.

11- Chuang, S.L., (1970), Investigation of Impact of Rigid and Elastic Bodies with Water, Management Information Services.

12- Nethercote, W.C.E., Mackay, M. and Menon, B., (1986), Some warship slamming investigations, D.R.E.A. Technical Memorandum 86/206.

13- Okada, S. and Sumi, Y., (2000), On the water impact and elastic response of a flat plate at small impact angles, Journal of Marine Science and Technology, Vol.5, p. 31-39. [DOI: 10.1007/s007730070019]

14- Ermanyuk, E.V. and Ohkusu, M., (2005), Impact of a disk on shallow water, Fluids and Structures,
Vol.20, p.

345-357.

[DOI:

10.1016/j.jfluidstructs.2004.10.002]

15- Huera-Huarte, F.J., Jeon, D. and Gharib, M., (2011), Experimental investigation of water slamming loads on panels, Ocean Engineering, Vol.38, p. 13471355.

16- Lewison, G.R.G. and Maclean, W.M., (1968), On the cushioning of water impact by entrapped air, Ship Res., Vo.12, p.116-130. [DOI: 10.1016/j.oceaneng.2011.06.004]

17- Lewison, G.R.G., (1970), On the reduction of slamming pressures, Trans. R.I.N.A., Vol. 112, p. $285-$ 306.

18- Asryan, N.G., (1972), Solid plate impact on surface of incompressible fluid in the presence of a gas layer between them, Izv. Akad. Nauk Arm. SSR Mekh, Vol. 25, p. 32-49.

19- Hicks, P. and Purvis, R., (2010), Air cushioning and bubble entrapment in three-dimensional droplet impacts, Fluid Mechanics, Vol. 649, p.135-163. [DOI: 10.1017/S0022112009994009]

20- Bouwhuis, W., van der Veen, R.C.A., Tran, T., Keij, D. L., Winkels, K.G., Peters, I.R., van der Meer, D., Sun, C., Snoeijer, J.H. and Lohse, D., (2012), Maximal Air Bubble Entrainment at Liquid-Drop Impact, PHYSICAL REVIEW LET TE RS, PRL 109, 264501. [DOI: 10.1103/PhysRevLett.109.264501] 21- Yousefnezhad, R. and Zeraatgar, H., (2014), A parametric study on water-entry of a twin wedge by boundary element method, J Mar Sci Technol, vol. 19, p. 314-326. [DOI: 10.1007/s00773-013-0250-1]

22- Katsikadelis, J.T., (2002), BOUNDARY ELEMENTS: Theory and Applications, Elsevier. 